J Chem Theory Comput. 2019 June 11; 15(6): 3623-3634. doi:10.1021/acs.jctc.9b00259.

\title{
Analytical Energy Gradients for the Cluster-in-Molecule MP2 Method and Its Application to Geometry Optimizations of Large Systems
}

\author{
Zhigang Ni†, ${ }^{\dagger}$, Yuqi Wang ${ }^{\dagger}$, Wei $\mathrm{Li}^{\dagger}$, Peter Pulay ${ }^{\star}, \ddagger$, Shuhua $\mathrm{Li}^{\star}{ }^{\star} \dagger$ \\ †School of Chemistry and Chemical Engineering, Key Laboratory of Mesoscopic Chemistry of \\ Ministry of Education, Institute of Theoretical and Computational Chemistry, Nanjing University, \\ Nanjing 210023, P. R. China \\ ‡Department of Chemistry and Biochemistry, University of Arkansas, Fayetteville, Arkansas \\ 72701, United States
}

\begin{abstract}
An efficient analytical energy gradient algorithm for the cluster-in-molecule (CIM) second order Møller-Plesset perturbation theory (MP2) method is presented. In our algorithm, the gradient contributions from the nonseparable term of the two-body density matrix on a given atom is extracted from calculations on a cluster constructed for this atom. The other terms in the CIMMP2 energy gradient expression are evaluated by constructing the density matrices of the whole system with the contributions from all clusters constructed. For basis sets with diffuse functions, tight CIM parameters are necessary to obtain accurate gradients. Benchmark calculations show that the CIM-MP2 method can accurately reproduce the conventional MP2 gradients and geometries for larger systems. The optimized structure of a 174-atom oligopeptide using the CIMMP2 method with the cc-pVDZ basis set is in good agreement with the corresponding crystal structure. The present CIM-MP2 gradient program can be used for optimizing the geometries of large systems with hundreds of atoms on ordinary workstations.
\end{abstract}

\section{Graphical Abstract}

\footnotetext{
*Corresponding Authors: Peter Pulay. pulay@uark.edu. Shuhua Li. shuhua@nju.edu.cn.

Supporting Information

The Supporting Information is available free of charge on the ACS Publications website at DOI: 10.1021/acs.jctc.9b00259.

Detailed analysis of the sparsity of the $\mathbf{X}, \mathbf{W}, \mathbf{Y}$, and $\overline{\mathbf{T}}$ matrices; numerical gradients; and structures of the hydrocarbons (PDF)

The authors declare no competing financial interest.
} 


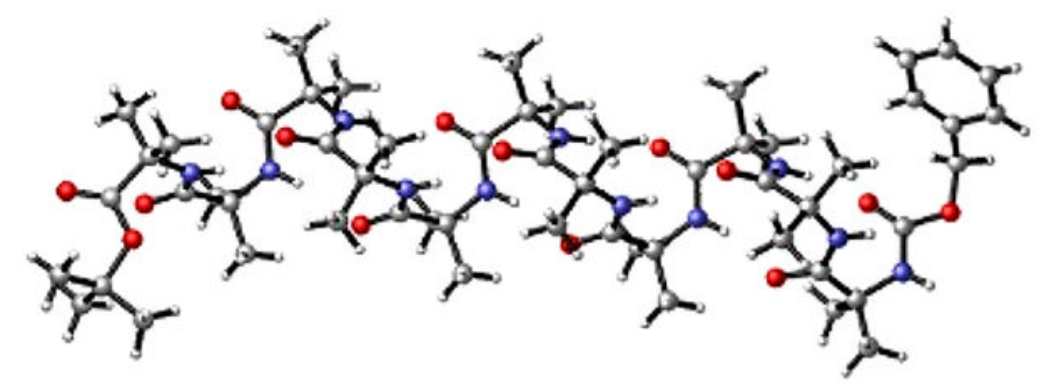

Geometry optimization using CIM-MP2/cc-pVDZ

174 atoms 1599 basis functions

\section{INTRODUCTION}

Analytical energy gradients are crucial for computing molecular properties using electronic structure methods. Electron correlation methods are needed for accurate prediction of molecular energies and geometries, particularly for systems where dispersion is important. However, traditional electron correlation methods are usually limited to small and mediumsized systems, because of their steep computational scaling with molecular size. Even the simplest traditional correlation method, second order Møller-Plesset perturbation theory (MP2), scales formally with the fifth power of the molecular size if the basis set quality is kept constant. The steep scaling of traditional electron correlation methods is due to their formulation in terms of delocalized canonical molecular orbitals (CMOs). Most traditional electron correlation methods are naturally orbital-invariant, i.e., unchanged under a unitary mixing of the occupied orbitals and a separate mixing of the virtual orbitals among themselves. Such methods include configuration interaction (CI) with various levels of substitution (singles, doubles, triples, etc.), e.g., CID, CISD, CISDT, and analogous coupled cluster (CC) techniques (CCD, CCSD, CCSDT).

It has been recognized that localized molecular orbitals (LMOs) can be used to greatly reduce the computational scaling of electron correlation methods. Local correlation methods were developed in the early 1980s by formulating the corresponding equations in terms of localized orbitals and eliminating insignificant contributions. ${ }^{1-5}$ Many-body perturbation methods, as usually formulated, are not orbital invariant, ${ }^{6}$ but they can be reformulated in terms of a nondiagonal zeroth order Hamiltonian to restore orbital invariance. ${ }^{7}$ Following the 1980s, a number of groups have developed various local correlation methods. ${ }^{8-37}$ These methods can be classified into two categories. The first type of local correlation methods $1,2,4,5,8-37$ treats the whole molecule at once but omits insignificant contributions, like pair correlation between distant occupied localized orbitals or substitution of an occupied orbital by a distant unoccupied orbital. The second class is based on transferring the results from smaller clusters (here clusters refers to a subset of localized orbitals) to the whole molecule. ${ }^{3,38-60}$ Compared to the first class, cluster-based methods can be applied to larger systems and are easier to parallelize because clusters can be treated independently. 
However, they involve more redundant computation. A recent review paper ${ }^{55}$ contains a detailed discussion of local correlation methods.

The cluster-in-molecule (CIM) method is an efficient local correlation approach for large systems which has been developed since $2002 .{ }^{46-54}$ As a method of the second class, CIM uses localized orbitals (both occupied and virtual) to divide a large molecule into overlapping clusters. Each cluster consists of a subset of occupied LMOs centered on one or a few atoms. The local virtual space is represented by a subset of atomic orbitals projected against the occupied space (PAOs). ${ }^{61}$ The CIM correlation energy is written as a sum over the correlated occupied orbitals. This is possible for virtually any correlation method. The correlation energy contribution of an occupied LMO is determined by electron correlation calculation on a cluster. Various levels of theory have been implemented within the CIM framework, including MP2, resolution of identity MP2 (RI-MP2), ${ }^{62}$ CCD, CCSD, CCSD with perturbative triples correction $[\operatorname{CCSD}(\mathrm{T})]$, and completely renormalized $\mathrm{CR}-\mathrm{CC}(2,3){ }^{63}$ The MP2 or CC equations can be solved within each cluster independently. In the latest CIM scheme, quasi-canonical molecular orbitals (QCMOs) ${ }^{48}$ are used to solve the excitation amplitudes at various levels of theory. As a result, the equations for MP2 amplitudes or the perturbative triples amplitudes can be solved in a noniterative manner, at the cost of extra orbital transformations. Recently, we reported a fully optimized implementation of the CIM method and its applications to several large chemical systems. ${ }^{56}$ Kállay and co-workers ${ }^{64-67}$ and Neese and co-workers ${ }^{68}$ developed promising methods by combining the CIM framework with other techniques.

Analytical MP2 energy gradients were first developed by the Pople group. ${ }^{69}$ The computational scaling of MP2 forces is the same as MP2 energy calculations. However, the calculation of MP2 forces is considerably more expensive than the MP2 energy, both in terms of CPU time and storage requirements. More effort is required for the solution of the coupled perturbed Hartree-Fock (CPHF) equations and the construction of the two-body density matrix, and larger disk space is needed for the storage of the amplitudes. Efficient MP2 gradient programs have been reported, ${ }^{7,69-80}$ but it is still difficult to calculate MP2 gradients for large systems.

In the past two decades, several analytical MP2 energy gradient methods within the local correlation framework have been proposed. For example, Werner and co-workers ${ }^{81}$ formulated the analytical energy gradient for the local MP2 (LMP2) method. Later, they extended it to the resolution of the identity LMP2 (RI-LMP2) method. ${ }^{82}$ Recently, Dornbach and Werner presented the LMP2 energy gradients using intrinsic bond orbitals (IBOs). ${ }^{83}$ Frank and co-workers implemented the gradient for MP2 based on the PNO approximation (PNO-MP2) ${ }^{84}$ Pinski and Neese reported the analytical gradient for the domain-based local pair natural orbital MP2 (DLPNO-MP2) ${ }^{85}$ For the cluster-based methods, Kjærgaard and co-workers derived the divide-expand-consolidate MP2 (DEC-MP2) gradient ${ }^{86}$ and DECRI-MP2 gradient. ${ }^{87}$ In addition, the MP2 energy gradient has also been implemented within several other fragmentation methods, which include the fragment molecular orbital (FMO) method, ${ }^{88,89}$ the systematic molecular fragmentation method, ${ }^{90}$ the molecular tailoring approach, ${ }^{91}$ the generalized energy-based fragmentation (GEBF) method, ${ }^{92}$ and the divideand-conquer (DC) method. ${ }^{93}$ 
In the present paper, we report analytical MP2 energy gradients within the CIM framework. We construct the effective relaxed two-body MP2 density matrix of the full system as the sum of contributions from clusters, and the final gradients of the system are computed by multiplying these density matrices with electron integral derivatives. However, for the gradient contributions from the nonseparable term of the two-body density matrix, we propose a single-cluster method to evaluate. This combined strategy is essential for evaluating the CIM-MP2 energy gradients when the diffuse basis functions are used. The accuracy of the CIM-MP2 gradients is assessed by comparing with the corresponding conventional MP2 gradients. The CIM-MP2 gradient is applied for geometry optimizations of several test molecules. Benchmark calculations show that the present CIM-MP2 gradient program reproduces the conventional MP2 gradients reasonably well and is reliable for geometry optimization of large systems.

\section{THEORY}

\subsection{Orbital-Invariant MP2 Energy Gradient.}

We use the orbital-invariant MP2 energy gradient formalism., ${ }^{7,79}$ This was applied successfully to LMP2. ${ }^{81}$ One of its advantages is that it does not require special formulation if only part of the orbital space (e.g., the valence orbitals) are correlated; the usual formulation leads to a very complicated formalism. ${ }^{80}$ The MP2 correlation energy can be expressed in orbital-invariant form as

$$
\Delta E_{\mathrm{MP} 2}=\sum_{i j}\left\langle\mathbf{K}^{i j}+\mathbf{R}^{i j} \mid \overline{\mathbf{T}}^{i j}\right\rangle
$$

where $\langle\mathbf{A}\rangle$ denotes the trace of matrix $\mathbf{A}$ and $\mathbf{R}$ is the MP2 residual given by

$$
\mathbf{R}^{i j}=\mathbf{K}^{i j}+\mathbf{F T}^{i j} \mathbf{S}+\mathbf{S} \mathbf{T}^{i j} \mathbf{F}-\mathbf{S} \sum_{k}\left[F_{i k} \mathbf{T}^{k j}+F_{k j} \mathbf{T}^{i k}\right] \mathbf{S}
$$

where $K_{a b}^{i j}=(a i \mid b j)$ is a two-electron integral in the Mulliken (charge density) notation, $\mathbf{F}$ is the Fock matrix, $\mathbf{S}$ is the overlap matrix, and $\left(\mathbf{T}^{i j}{ }_{a b}\right.$ denotes the amplitude for the substitution from an occupied orbital pair $i j$ to virtual orbitals $a b$. For the correct MP2 solution, the components of the residual matrix must vanish in the virtual subspace. The $\mathbf{F}$ and $\mathbf{S}$ matrices should refer to the same basis as the amplitude matrix. The normalized contravariant amplitude matrix ${ }^{94} \overline{\mathbf{T}}$ is defined as

$$
\overline{\mathbf{T}}^{j i}=2 \mathbf{T}^{i j}-\mathbf{T}^{j i}
$$

The gradient of the MP2 energy can be written in the AO basis as

$$
\Delta E_{\mathrm{MP} 2}^{x}=\sum_{\mu \nu} X_{\mu \nu}^{\prime} h_{\mu \nu}^{x}+\sum_{\mu \nu} W_{\mu \nu}^{\prime} S_{\mu \nu}^{x}+\sum_{\mu \nu \lambda \sigma} \Gamma_{\mu \nu, \lambda \sigma}(\mu \nu \mid \lambda \sigma)^{x}
$$

where the superscript $x$ represents the derivative with respect to one of the nuclear coordinates. $\mathbf{h}$ and $\mathbf{S}$ are the core Hamiltonian and overlap matrices. $(\mu \nu \mid \lambda \sigma)$ is an AO-based 
two-electron integral in the Mulliken (charge density) notation. All three terms in eq 4 have the same structure: the trace of the product of a density matrix $\left(\mathbf{X}^{\prime}, \mathbf{W}^{\prime}\right.$, and $\left.\Gamma\right)$ with an integral derivative matrix. The explicit form of the $\mathbf{X}^{\prime}$ matrix is

$$
\begin{aligned}
& \mathbf{X}^{\prime}=\mathbf{X}+\mathbf{Z} \\
& =2 \mathbf{C}_{\mathrm{v}} \mathbf{X}_{1} \mathbf{C}_{\mathrm{v}}^{\dagger}-2 \mathbf{C}_{\mathrm{o}} \mathbf{A} \mathbf{C}_{\mathrm{o}}^{\dagger}+\mathbf{Z}
\end{aligned}
$$

with

$$
\mathbf{X}_{1}=\sum_{i j} \overline{\mathbf{T}}^{j i} \mathbf{S T}^{i j}
$$

and

$$
A_{i k}=\sum_{j}\left\langle S T^{j k} S T^{i j}\right\rangle
$$

where $\mathbf{C}_{\mathrm{o}}$ and $\mathbf{C}_{\mathrm{v}}$ denote the coefficient matrices of occupied and virtual orbitals. The $\mathbf{Z}$ matrix is the solution of the CPHF equations using the z-vector method of Handy and Schaefer. ${ }^{95}$

The $\mathbf{W}^{\prime}$ matrix is expressed as

$$
\mathbf{W}^{\prime}=\mathbf{W}-\frac{1}{4} \mathbf{D Y} \mathbf{C}_{\mathrm{o}}^{\dagger}-\widetilde{\mathbf{Z}}-\frac{1}{2} \mathbf{D G}(\mathbf{Z}) \mathbf{D}
$$

with

$$
W_{\mu \nu}=2\left[\sum_{i j} \overline{\mathbf{T}}^{j i} \mathbf{F} \mathbf{T}^{i j}-\sum_{i j} \sum_{k}\left(F_{i k} \mathbf{T}^{k j} \mathbf{S} \overline{\mathbf{T}}^{j i}+F_{k i} \mathbf{T}^{j k} \mathbf{S} \overline{\mathbf{T}}^{i j}\right)-2 \sum_{i j} \mathbf{C}_{\mathrm{v}} \overline{\mathbf{T}}_{\mathrm{vv}}^{j i} \mathbf{K}_{\mathrm{vo}}^{i j} \mathbf{C}_{\mathrm{o}}^{\dagger}\right]_{\mu \nu}
$$

and

$$
\begin{gathered}
Y_{\nu i}=-4\left[\mathbf{S C}_{\mathrm{v}} \sum_{i j} \overline{\mathbf{T}}_{\mathrm{vv}}^{i j} \mathbf{K}_{\mathrm{vo}}^{i j}\right]_{v i}+4 \sum_{j \mu \lambda}(\mu \nu \mid \lambda j) \bar{T}_{i j}^{\lambda \mu}+4\left[\mathbf{G}(\mathbf{X}) \mathbf{C}_{\mathrm{o}}-\mathbf{F} \mathbf{C}_{\mathrm{o}} \mathbf{A}\right]_{v i} \\
\widetilde{\mathbf{Z}}=\frac{1}{2}\left(\mathbf{C}_{\mathrm{v}} \tilde{z} \mathbf{C}_{\mathrm{o}}^{\dagger}+\mathbf{C}_{\mathrm{o}} \tilde{z}^{\dagger} \mathbf{C}_{\mathrm{v}}^{\dagger}\right) \quad \tilde{z}_{a i}=\varepsilon_{i} z_{a i}
\end{gathered}
$$

where $\mathbf{D}$ is the HF density matrix, the subscripts vv and vo represent the virtual-virtual and virtual-occupied blocks of corresponding matrices, and $\boldsymbol{\varepsilon}$ is the orbital energy. All quantities without subscripts in eq 9 are in the $\mathrm{AO}$ basis. The notation $\mathbf{G}(\mathbf{Q})_{\mu \nu}$ is defined as

$$
\mathbf{G}(\mathbf{Q})_{\mu \nu}=\sum_{\lambda \sigma}\left\{(\mu \nu \mid \lambda \sigma)-\frac{1}{2}(\mu \lambda \mid \sigma \nu)\right\} \mathbf{Q}_{\lambda \sigma}
$$


The two-body density matrix $\Gamma$ is expressed as the sum of a nonseparable (NS) part $\Gamma^{\mathrm{NS}}$ and a separable (S) part $\Gamma^{\mathrm{S}}$ :

$$
\Gamma_{\mu \nu, \lambda \sigma}=\underbrace{2 \bar{T}_{\sigma \nu}^{\mu \lambda}}_{\Gamma^{\mathrm{NS}}}+\underbrace{D_{\lambda \sigma} X_{\mu \nu}^{\prime}-\frac{1}{2} D_{\nu \sigma} X_{\mu \lambda}^{\prime}}_{\Gamma}
$$

The gradient of the HF energy also has three terms, similar to eq 4 just with different density matrices. Thus, the final MP2 energy gradient can be calculated by

$$
\begin{aligned}
& E_{\mathrm{MP} 2}^{x}=E_{\mathrm{HF}}^{x}+\Delta E_{\mathrm{MP} 2}^{x} \\
& =\sum_{\mu \nu}\left(X_{\mu \nu}^{\prime} \mathrm{HF}+X_{\mu \nu}^{\prime \mathrm{MP} 2}\right) h_{\mu \nu}^{x}+\sum_{\mu \nu}\left(W_{\mu \nu}^{\prime} \mathrm{HF}+W_{\mu \nu}^{\prime \mathrm{MP} 2}\right) S_{\mu \nu}^{x}+\sum_{\mu \nu \lambda \sigma}\left(\Gamma_{\mu \nu, \lambda \sigma}^{\mathrm{HF}}+\Gamma_{\mu \nu, \lambda \sigma}^{\mathrm{MP} 2}\right)(\mu \nu \\
& \lambda \sigma) x
\end{aligned}
$$

The expression for the HF density matrix is well-known ${ }^{69,79}$ and not reproduced here.

\subsection{CIM-MP2 Energy.}

The MP2 correlation energy of a system can be expressed as the sum of contributions from all the occupied orbitals:

$$
\Delta E_{\mathrm{MP} 2}=\sum_{i}^{N_{\mathrm{occ}}} \Delta E_{i}
$$

When MP2 residuals are zero, eq 1 can be simplified as

$$
\Delta E_{\mathrm{MP} 2}=\sum_{i j}\left\langle\mathbf{K}^{i j} \overline{\mathbf{T}}^{j i}\right\rangle=\sum_{i j, a b} K_{a b}^{i j} \bar{T}_{a b}^{j i}
$$

The contribution of a given occupied orbital $i$ can be expressed as

$$
\Delta E_{i}=\sum_{j, a b} K_{a b}^{i j} \bar{T}_{a b}^{j i}
$$

In the CIM framework, the correlation energy contribution of an occupied orbital $i$ is obtained within a cluster, which contains orbital $i$ itself, its spatially neighboring occupied orbitals, and a subset of virtual orbitals distributing over a certain region. We now describe how to construct such a cluster.

In the CIM framework, we first perform an HF calculation for the full system and the occupied LMOs are obtained by the Boys ${ }^{96,97}$ (or Pipek-Mezey ${ }^{98}$ ) localization method. Each occupied LMO is assigned to an atom, which has the largest absolute Mulliken charge, and this atom is called the central atom of the occupied LMO. Several LMOs may be centered on one atom. The distance between two occupied LMOs is defined as the distance 
between their central atoms. Occupied LMOs centered on one atom (called as central orbitals) and their spatially neighboring occupied LMOs (called as buffer orbitals) within a distance threshold $\zeta$ are combined as the MO domain [I] of a cluster. The PAOs centered on the atoms in the MO domain are chosen as the virtual space of the cluster.

Next, a suitable AO domain $\Omega(I)$, which contains only part of the basis functions of the full system, is determined for the MO domain. We have recently developed an improved algorithm for constructing the virtual LMOs and the AO domain. ${ }^{56}$ By employing the Boughton-Pulay (BP) projection method, ${ }^{61}$ the occupied LMOs and PAOs within a cluster are expressed as the linear combinations of the basis functions in the AO domain,

$$
\begin{gathered}
\phi_{i^{\prime}}=\sum_{\mu \in \Omega(I)} C_{\mu i^{\prime}}\left|\chi_{\mu}\right\rangle \\
\tilde{\chi}_{a^{\prime}}=\sum_{\mu \in \Omega(I)} C_{\mu a^{\prime}}\left|\chi_{\mu}\right\rangle
\end{gathered}
$$

where $\varphi_{i}^{\prime}$ and $\tilde{\chi}_{a^{\prime}}$ represent the truncated occupied LMOs and PAOs in the cluster, respectively. The redundancy of the truncated PAOs within a cluster is eliminated by the canonical orthogonalization procedure, which leads to a set of orthonormal virtual orbitals for the cluster.

In summary, a CIM cluster consists of a subset of occupied and virtual LMOs, and these orbitals are approximately represented with the basis functions in the AO domain of the cluster. Another parameter that controls the size of the AO domain is denoted as $\eta$ in our previous work. ${ }^{56} \eta$ is the BP projection threshold used during the determination of the AO domain. A smaller $\eta$ value gives a larger AO domain. Therefore, in current CIM approach, the accuracy is controlled by two parameters: the distance threshold $\zeta$ and projection threshold $\eta$. A larger $\zeta$ value or a smaller $\eta$ value leads to more accurate results. The default values are set as $\zeta=5.5 \AA$ and $\eta=0.05$. For simplicity, in the following context, we use a pair of numbers, like 5.5/0.05, to denote the two parameters. With these defaults, the CIM approach can recover $99.9 \%$ of the conventional correlation energy for most systems.

To avoid an iterative solution of the MP2 equations, the LMOs are transformed to QCMOs which are delocalized within the cluster. The occupied (or virtual) QCMOs are linear combinations of occupied (or virtual) LMOs within a cluster. The expansion coefficients are determined by diagonalizing the LMO representation of the Fock matrix within the cluster:

$$
\mathbf{F U}=\mathbf{U} \varepsilon
$$

where

$$
F_{i^{\prime} j^{\prime}}=\sum_{u, v \in \Omega(I)} C_{u i^{\prime}}^{\prime} F_{u v} C_{v j^{\prime}}^{\prime}
$$

and 


$$
\psi_{i}=\sum_{k^{\prime} \in \Omega(I)} U_{k^{\prime} i} \phi_{k^{\prime}}
$$

where $\Psi_{i}$ are the QCMOs.

After solving the MP2 equation using QCMOs, the energy contribution from the occupied orbitals can be calculated in cluster $[P]$ as

$$
\Delta E_{i^{\prime} \in[P]}=\sum_{j, a b \in[P]} \widehat{K}_{a b}^{i^{\prime} j} \widehat{\bar{T}}_{a b}^{j i^{\prime}}
$$

where one of the indices of $\mathbf{K}$ and $\overline{\mathbf{T}}$ matrices is transformed back from QCMO to LMO space by

$$
\begin{aligned}
& \widehat{K}_{a b}^{i^{\prime} j}=\sum_{i \in[P]} U_{i i^{\dagger}}^{\dagger} K_{a b}^{i j} \\
& \widehat{\bar{T}}_{a b}^{i^{\prime} j}=\sum_{i \in[P]} U_{i i^{\prime}}^{\dagger} \bar{T}_{a b}^{i j}
\end{aligned}
$$

\subsection{MP2 Gradient within the CIM Framework.}

In the present CIM-MP2 gradient algorithm, we attempt to obtain the density matrices in eq 4 for the full system as a sum of the contributions from the central orbitals within clusters. Within each cluster, all quantities are first calculated using QCMOs and then transformed to LMOs to obtain the contributions of central orbitals. In this subsection, we use the labels $i, j$, $\ldots$ and $a, b, \ldots$ to denote the occupied and virtual QCMOs, respectively. Primed lower case letters denote LMOs.

The $\mathbf{X}^{\prime}$ matrix is the sum of the $\mathbf{X}$ matrix and the $\mathbf{Z}$ matrix. The $\mathbf{Z}$ matrix is the solution of CPHF equation, and it will be discussed later. The first contribution of the $\mathbf{X}$ matrix is the $\mathbf{X}_{1}$ matrix of eq 6. $\mathbf{X}_{1}$ is first calculated in MO basis and then transformed to AO basis. Within a CIM cluster, virtual orbitals are orthogonal to each other and $\mathbf{S}$ is a unit matrix. Thus, the $\mathbf{X}_{1}$ matrix in cluster $[P]$ can be expressed as

$$
\mathbf{X}_{1}=\sum_{i j \in[P]} \overline{\mathbf{T}}^{j i} \mathbf{T}^{i j}
$$

A part of the $\mathbf{X}_{1}$ matrix, which includes only contributions from the central orbitals, can be formulated as

$$
\widehat{\mathbf{X}}_{1}=\sum_{i^{\prime}}^{\text {cen }} \sum_{j \in[P]} \widehat{\overline{\mathbf{T}}}^{j i^{\prime}} \widehat{\mathbf{T}}^{i^{\prime} j}
$$

where the $\mathbf{T}$ and $\overline{\mathbf{T}}$ matrices are in virtual MO basis and one of the occupied orbital indices is transformed from QCMO to LMO by eq 25 and 


$$
\widehat{\bar{T}}_{a b}^{i^{\prime} j}=\sum_{i \in[P]} U_{i i^{\dagger}}^{\dagger} \bar{T}_{a b}^{i j}
$$

At last, the $\widehat{\mathbf{x}}_{1}$ matrix is transformed to the AO basis by $\mathbf{C}_{\mathrm{v}} \widehat{\mathbf{x}}_{1} \mathbf{C}_{\mathrm{v}}^{\dagger}$.

The second contribution of the $\mathbf{X}$ matrix is the $\mathbf{A}$ matrix of eq 7. To obtain the contributions of the central orbitals, one of the indices of the A matrix is transformed from QCMO to LMO basis:

$$
\widehat{A}_{i k^{\prime}}=\sum_{k \in[P]} U_{k k^{\prime}}^{\dagger} A_{i k}
$$

Note that $\widehat{\mathbf{A}}$ is a $\left(N_{\mathrm{OCC}}^{P} \times N_{\text {cen }}^{P}\right)$ matrix where $N_{\mathrm{OCC}}^{P}$ and $N_{\text {cen }}^{P}$ refer to the number of occupied and central orbitals in cluster $[P]$. Similarly to the $\widehat{\mathbf{x}}_{1}$ matrix, $\widehat{\mathbf{A}}$ is also transformed to the AO basis by $\mathbf{C}_{\mathrm{o}} \widehat{\mathbf{A}} \mathbf{C}_{\mathrm{cen}}^{\dagger}$, where $\mathbf{C}_{\mathrm{cen}}$ is the coefficient matrix of central LMOs.

The first contribution to the $\mathbf{W}^{\prime}$ matrix is the $\mathbf{W}$ matrix, eq 9. All three of its terms have the same structure as the $\mathbf{X}$ matrix; hence, they are treated in a similar way to obtain the contributions of central orbitals. Since a cluster contains only part of the basis functions of the full system, there is a correspondence between basis functions in a cluster and in the full system. The $\mathbf{X}$ and $\mathbf{W}$ matrices of the clusters correspond to blocks of the $\mathbf{X}$ and $\mathbf{W}$ matrices of the full system. The $\mathbf{X}$ and $\mathbf{W}$ matrices of the full system are constructed by summing the corresponding $\mathbf{X}$ and $\mathbf{W}$ blocks of clusters. The data in the Supporting Information (SI) show that the matrix elements of $\mathbf{X}$ and $\mathbf{W}$ decrease quickly when two basis functions correspond to two distant atoms. This indicates that the $\mathbf{X}$ and $\mathbf{W}$ matrices constructed with the quantities from clusters are good approximations to the corresponding matrices of the full system.

The second contribution to the $\mathbf{W}^{\prime}$ matrix is related to the $\mathbf{Y}$ matrix, eq $10 . \mathbf{Y}$ is also used in the $z$-vector method to avoid the solution of the CPHF equations for all perturbations. In the current CIM-MP2 gradient algorithm, we solve the CPHF equation for the full system; thus, we need the full $\mathbf{Y}$ matrix with the dimension of $N_{\text {bas }} \times N_{\text {occ }}$, where $N_{\text {bas }}$ and $N_{\text {occ }}$ denote the number of basis functions and occupied orbitals of the full system. Within a cluster $[P]$, $\mathbf{Y}$ is a $\left(N_{\mathrm{bas}}^{\mathrm{p}} \times N_{\mathrm{OCC}}^{p}\right)$ matrix where $N_{\mathrm{bas}}^{\mathrm{p}}$ is the number of basis functions of cluster [ $P$ ]. The $\widehat{\mathbf{Y}}$ matrix in the central LMO basis is obtained by

$$
\widehat{Y}_{\nu i^{\prime}}=\sum_{i \in[P]} U_{i i^{\prime}}^{\dagger} Y_{v i}
$$

In the following context, we will use $\mathbf{Y}^{\mathrm{LMO}}$ and $\mathbf{Y}^{\mathrm{CMO}}$ to denote the $\mathbf{Y}$ matrix in LMO basis and CMO basis, respectively. In the full system, each occupied LMO is treated as a central orbital within a certain cluster. The $\mathbf{Y}^{\mathrm{LMO}}$ matrix of the full system can be obtained by 
collecting the $\widehat{\mathbf{Y}}$ matrices from all clusters. In the SI, we have compared the similarity of the $\widehat{\mathbf{Y}}$ matrix obtained from various clusters and the corresponding $\mathbf{Y}^{\mathrm{LMO}}$ matrix of the full system. The $\mathbf{Y}^{\mathrm{LMO}}$ matrices for four LMOs are randomly selected for comparison. The corresponding two matrices are very close to each other, with the largest deviation of $7.36 \times$ $10^{-5}$. Thus, the $\widehat{\mathbf{Y}}$ matrix from all clusters is a good approximation of the corresponding $\mathbf{Y}^{\mathrm{LMO}}$ matrix. The $\mathbf{Y}^{\mathrm{CMO}}$ matrix is then computed by

$$
\mathbf{Y}^{\mathrm{CMO}}=\mathbf{Y}^{\mathrm{LMO}} \widetilde{\mathbf{U}}
$$

where $\tilde{\mathrm{U}}$ is the unitary transformation matrix between LMOs and CMOs of the full system. With the $\mathbf{Y}^{\mathrm{CMO}}$ matrix, the second term in eq 8 can be calculated.

As mentioned above, the $\mathbf{Y}^{\mathrm{CMO}}$ matrix is also used in the CPHF equations. Since the latter are solved in the full system, the $\mathbf{Z}$ and $\widetilde{\mathbf{Z}}$ matrices are calculated in a similar way as in the conventional method.

It should be mentioned that we do not solve the coupled-perturbed localization (CPL) equations as described in refs 81-84. In the CIM approach, a cluster is a sufficiently large correlation domain to obtain the correlation energy contributions of the central orbitals. With the $\mathbf{Y}^{\mathrm{LMO}}$ matrix obtained above, we have found that the $\mathbf{u}$ matrix in eq 41 in ref 81 is very close to $O$, and thus the related $\mathbf{v}$ term (in eqs 46 and 47 in ref 81 ) can be neglected. In this case, it is not necessary to solve the CPL equations in the CIM approach.

In Table 1, we compare the gradient contributions of each term in the final gradient expression from the CIM approach with that from the conventional MP2 using system (7) in Figure 1. The signed maximum deviation (in $\mathrm{m} E_{\mathrm{h}} / \mathrm{a}_{0}$ ) of each term is listed in Table 1. For comparison, we use two basis sets, 6-31G(d) and 6-31+G. For 6-31G(d), two set of CIM parameters, 5.5/0.05 and 8.0/0.01, are used, and for 6-31+G, an additional set of parameters, $10.0 / 0.01$, is used. Table 1 shows that when the basis set contains no diffuse functions, the final gradient contributions from $\mathbf{X}^{\prime}$ and $\mathbf{W}^{\prime}$ matrices calculated by the CIM approach have only small deviations from conventional results. In the diffuse basis set case, the errors are significantly larger. For both basis sets, the deviations get smaller by tightening the thresholds.

At this stage, we have described how to obtain the $\mathbf{X}^{\prime}$ and $\mathbf{W}^{\prime}$ matrices in the CIM framework. Next, we will discuss how to evaluate the $\overline{\mathbf{T}}$ matrix in eq 13, which is the NS term of the two-body density matrix $\Gamma$. In the conventional algorithm, the final $\overline{\mathbf{T}}$ matrix in the AO basis is obtained via the following chain of transformation:

$$
\bar{T}_{a b}^{i j} \stackrel{\overline{\mathbf{T}}_{\mathrm{AO}}=\mathbf{C}_{\mathrm{v}} \overline{\mathbf{T}}_{\mathrm{MO}} \mathbf{C}_{\mathrm{v}}^{\dagger}}{\longrightarrow} \bar{T}_{\mu \lambda}^{i j} \stackrel{\text { Yoshimine bin sort }}{\longrightarrow} \bar{T}_{i j}^{\mu \lambda} \stackrel{\overline{\mathbf{T}}_{\mathrm{AO}}^{\mu \lambda}=\mathbf{C}_{\mathrm{o}} \overline{\mathbf{T}}_{\mathrm{MO}}^{\mu \lambda} \mathbf{C}_{\mathrm{o}}^{\dagger}}{\longrightarrow} \bar{T}_{\nu \sigma}^{\mu \lambda}
$$

In the CIM framework, the $\overline{\mathbf{T}}$ matrix within a cluster can be treated as

$$
\bar{T}_{a b}^{i j} \stackrel{\overline{\mathbf{T}}_{\mathrm{AO}}=\mathbf{C}_{\mathrm{v}} \overline{\mathbf{T}}_{\mathrm{MO}} \mathbf{C}_{\mathrm{v}}^{\dagger}}{\longrightarrow} \bar{T}_{\mu \lambda}^{i j} \stackrel{\text { Yoshimine bin sort }}{\longrightarrow} \bar{T}_{i j}^{\mu \lambda} \stackrel{\overline{\mathbf{T}}_{\mathrm{LMO}}^{\mu \lambda}=\mathbf{U} \overline{\mathbf{T}}_{\mathrm{QCMO}}^{\mu} \mathbf{U}^{\dagger}}{\longrightarrow} \widehat{\bar{T}}_{i^{\prime} j^{\prime}}^{\mu \lambda}
$$


The $\widehat{\overline{\mathbf{T}}}$ matrix is in LMO basis. To consider only the contributions from central orbitals, the first index of the $\widehat{\overline{\mathbf{T}}}$ matrix can be restricted to the central orbital. Thus, the $\widehat{\overline{\mathbf{T}}}$ matrix for $\mu \lambda$ pair has the dimension of $N_{\text {cen }}^{\mathrm{p}} \times N_{\text {OCC }}^{p}$. The $\overline{\mathbf{T}}$ matrix of the full system in LMO basis is constructed by combining the $\widehat{\overline{\mathbf{T}}}$ matrices of clusters. The $\overline{\mathbf{T}}$ matrix in AO basis is then obtained by

$$
\overline{\mathbf{T}}_{\mathrm{AO}}^{\mu \lambda}=\mathbf{L}_{\mathrm{o}} \overline{\mathbf{T}}_{\mathrm{LMO}}^{\mu \lambda} \mathbf{L}_{\mathrm{o}}^{\dagger}
$$

where $\mathbf{L}_{\mathrm{o}}$ is the coefficient matrix of occupied LMOs. Thus, the gradient contribution from the NS on a given atom is obtained from combining the results from all clusters, and this approach may be considered as the cluster-averaged method.

Table 1 shows that the deviation of the NS term from the CIM approach (relative to the conventional result) is quite small at the $6-31 \mathrm{G}(\mathrm{d})$ basis set. However, the deviation of this term (which is the dominant term in the total gradient) is very large even with tight thresholds (8.0/0.01) at the $6-31+\mathrm{G}$ basis set. To understand this large error, we have analyzed the sparsity of the AO-based $\overline{\mathbf{T}}$ matrix. When the basis set contains diffuse functions (such as 6-31+G), the matrix element $\bar{T}_{\nu \sigma}^{\mu \lambda}$ is non-negligible even when $\mu$ and $\lambda$ are centered on two distant atoms (say $10 \AA$ ). In the CIM framework, the matrix elements $\bar{T}_{\nu \sigma}^{\mu \lambda}$ for those distant atom pairs outside a cluster are all assumed to be zero. Thus, for basis sets with diffuse functions, tight CIM thresholds should be used to make the clusters larger so that a better approximation to the $\overline{\mathbf{T}}$ matrix can be obtained (see SI Section 1.3 for detailed analysis).

We propose a different algorithm for evaluating this term. In the new algorithm, for a cluster we use the full QCMO space directly to obtain the final gradients on atoms of this cluster. No transformation from QCMO to LMO is employed. QCMOs are directly transformed to AO basis via the chain of transformation (eq 32). The gradient contribution from the NS term in the cluster is calculated by

$$
\Delta E_{\mathrm{MP} 2}^{x \in[P]} \leftarrow \sum_{\mu \nu \lambda \sigma \in[P]} \bar{T}_{\nu \sigma}^{\mu \lambda}(\mu \nu \mid \lambda \sigma)^{x}
$$

Only the gradients on the central atoms (corresponding to the central orbitals) are extracted. This approach may be denoted as the single-cluster method. In some cases, an atom $A$ may be never assigned as a central atom. If this atom is spatially close to a central atom $B$, the gradient contribution from the NS term for the atom $A$ is obtained in the cluster centered on the atom $B$ (as a central atom).

For system (7) in Figure 1, we have compared the accuracy of the two methods in evaluating the gradient contributions from the NS term. The $6-31+\mathrm{G}$ basis set is used, and CIM parameters are set to $8.0 / 0.01$. Table 2 lists the deviation (in $\mathrm{m} E_{\mathrm{h}} / \mathrm{a}_{0}$ ) of the gradient contributions of the NS term by these two methods with respect to the results from the conventional method. Only the $x$-components of the forces on the first $10 \mathrm{C}$ atoms are 
compared. It can be seen from Table 2 that the single-cluster method is much more accurate than the cluster-averaged method. In the single-cluster method, only gradients on the central atoms are thought to be transferrable to the full system. This is consistent with the basic idea of the CIM approach. It should be pointed out that the method of expressing the density matrix of the full system as the sum of contributions from clusters is similar to the idea used in the energy gradient of the DC-MP2 method by Nakai and coworkers. ${ }^{93}$ The CIM-MP2 energy gradient evaluated in this way may be considered as an approximate derivative of the CIM-MP2 energy.

\subsection{Implementation.}

The present CIM-MP2 gradient algorithm has been implemented in the PQS package. ${ }^{99}$ Since parallelization is not implemented in the conventional MP2 gradient module of PQS, parallel calculations for individual CIM clusters are not available. However, calculations on different CIM clusters can be done independently. After calculating the contributions of the $\mathbf{X}, \mathbf{W}$, and $\mathbf{Y}$ matrices and the gradient contributions of the NS term in the clusters, the CPHF equation and the final gradient (except the contributions from the NS term) are calculated within the full system by eq 4 .

\section{RESULTS AND DISCUSSION}

\subsection{Benchmark Calculations of Energy Gradients.}

To show the accuracy of the present CIM-MP2 gradient approach, we have tested several systems with various basis sets. These systems include an amine macrocycle compound $\left(\mathrm{C}_{58} \mathrm{H}_{62} \mathrm{~N}_{6} \mathrm{O}_{4}\right)$, a poly(benzyl ether) dendrimer $\left(\mathrm{C}_{33} \mathrm{H}_{36} \mathrm{O}_{3}\right)$, a $\mathrm{CO}_{2}$ cluster $\left[\left(\mathrm{CO}_{2}\right)_{30}\right]$, a reaction complex with the phosphoric acid catalyst $\left(\mathrm{C}_{61} \mathrm{H}_{71} \mathrm{O}_{7} \mathrm{P}\right)$, the Cucurbit[6]uril molecule $\left(\mathrm{C}_{36} \mathrm{H}_{36} \mathrm{~N}_{24} \mathrm{O}_{12}\right)$, a short peptide $\left(\mathrm{C}_{24} \mathrm{H}_{42} \mathrm{~N}_{8} \mathrm{O}_{10}\right)$, the U-DPy molecule $\left(\mathrm{C}_{23} \mathrm{H}_{31} \mathrm{~N}_{5} \mathrm{O}_{4}\right)$, the hydroxysqualene molecule $\left(\mathrm{C}_{30} \mathrm{H}_{50} \mathrm{O}\right)$, and stearic acid $\left(\mathrm{C}_{18} \mathrm{H}_{36} \mathrm{O}_{2}\right)$. The structures are shown as systems (1)-(9) in Figure 1. For basis sets without diffuse functions, the parameters 5.5/0.05 are adopted, and for basis sets with diffuse functions, the parameters are set as 8.0/0.01. Table 3 compares the CIM-MP2 gradients with the results from the conventional MP2 method.

One can see from Table 3 that the present CIM-MP2 gradients are good approximations to the corresponding conventional MP2 gradients. For systems at various basis sets, especially when the system contains some delocalized groups, like an aromatic ring, the mean absolute deviation is still satisfactory, with the maximum deviation being about 0.003 atomic units.

As pointed out above, the present CIM-MP2 gradient is an approximate CIM-MP2 energy derivative. Therefore, it is worthwhile to compare the present analytical CIM-MP2 gradients with the corresponding numerical CIM-MP2 energy gradients for several systems. The numerical gradients were evaluated using the three-point central difference formula with a step size of $0.005 \AA$. The computational details are listed in SI Section II. The maximum deviation of the analytical CIM-MP2 gradients with respect to the numerical results is about $3 \mathrm{~m} E_{\mathrm{h}} / \mathrm{a}_{0}$, which is of the same order as the errors between analytical CIM-MP2 gradients 
and conventional MP2 gradients. This is understandable because the numerical CIM-MP2 gradients are close to the conventional analytical MP2 gradients.

We have also evaluated the scaling of the present CIM-MP2 gradient program for a series of linear hydrocarbons (see Figure S4) with different numbers of carbon atoms. The calculations were performed on a single core of a node equipped with two 12-core Intel Xeon Gold $6126 \mathrm{CPU}(2.60 \mathrm{GHz})$. The CPU times for CIM-MP2 (including all steps of gradient calculations) and conventional MP2 gradient, plotted in Figure 2, show that the crossover lies at systems with about 35 carbon atoms. In the present algorithm, the CPHF equations are solved in the full system. In our implementation, it is done in an integral-direct manner, and it only makes a small fraction of the total CPU time. For example, for the largest system in Figure 2, the total CPU time is $1558 \mathrm{~min}$ and the solution of CPHF equations takes only $57 \mathrm{~min}$. In addition, since the density matrices are multiplied with integral derivatives in the full system, the present CIM-MP2 gradient approach is not truly linear-scaling. However, it has a much lower scaling than the conventional MP2 gradient. The most time-consuming step in eq 4 is the two-electron term. As discussed above, the first term in eq 13 is calculated within clusters and the other two terms are calculated within the full system. It should be noted that for the gradient contributions from the NS term calculated within the clusters, we only need to calculate gradients on the central atoms. Usually there are only a few central atoms within a cluster. We only need to calculate the derivatives for two-electron integrals which contain basis functions on one of the central atoms.

To make the present CIM-MP2 gradient program more practical, we have parallelized the most time-consuming step, the two-electron term which is calculated within the full system. The parallelization is over the contracted shells. The parallel speedup is defined as

$$
S(p)=\frac{t(1)}{t(p)}
$$

where $t(1)$ and $t(p)$ are the elapsed times for running on 1 and $p$ processes. The speedup for $\mathrm{C}_{80} \mathrm{H}_{108}$ is shown in Figure 3. It exceeds an order of magnitude for this modest workstation.

With the parallelized CIM-MP2 gradient program, systems with thousands of basis functions can be calculated on a moderate workstation.

\subsection{Validation of the CIM-MP2 Gradients for Geometry Optimization.}

To demonstrate that the present CIM-MP2 gradients can be used for geometry optimization, we have randomly picked up five structures of system (7) in Figure 1 from the B3LYP/ccpVDZ optimization trajectory, and compared the CIM-MP2 gradients with the conventional MP2 gradients for these five structures at the def2-SVP basis set. The results are listed in Table 4. One can see that for all five structures, CIM-MP2 can reproduce the conventional MP2 gradients quite well. Thus the CIM-MP2 gradient is expected to be reliable for geometry optimization.

The CIM-MP2 gradients have been applied for geometry optimization of two medium-sized molecules. Our program is interfaced with Gaussian $16^{100}$ for geometry optimization. We 
have tested systems (7) and (8) in Figure 1, and compared our results with those from the conventional MP2 method. The root-mean-square deviation (RMSD) of the bond lengths of the structures from the CIM-MP2 and conventional MP2 methods, the converged total electronic energies of the two methods, and the CPU times are listed in Table 5.

The structures of the two systems optimized by the CIM-MP2 and conventional MP2 methods are shown in Figure 4. The RMSDs of the bond lengths are 0.0003 and $0.0004 \AA$, respectively. These results indicate that the CIM-MP2 method can reproduce the conventional MP2 geometry for the two systems. The CIM-MP2 electronic energies of the two systems at their optimized structures are also in good agreement with those from the conventional MP2 method. The CIM-MP2 optimization requires somewhat more steps to converge than the conventional MP2 optimization. This issue may result from the fact that the CIM-MP2 potential energy surface is slightly less smooth than the conventional MP2 potential energy surface. During the CIM-MP2 optimization, one may need to use a smaller step size when the structure is close to the local minimum. These benchmark calculations suggest that the present CIM-MP2 approach is reliable for geometry optimizations of large systems.

\subsection{Optimized Structure of an Oligopeptide.}

In this subsection, we have applied CIM-MP2 to obtain the optimized structure for an oligopeptide, $\mathrm{Z}-(\mathrm{Aib})_{11}-\mathrm{O} t \mathrm{Bu}$, at the cc-pVDZ basis set. The crystal structure of this

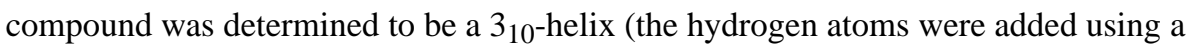
theoretical riding model approach) ${ }^{101}$ and is shown in Figure 5. This system has 174 atoms and 1599 basis functions. It is very demanding to perform geometry optimization with the conventional MP2 method for such a large system. For comparison, we have also optimized the structure with the density functional theory (DFT). Two functionals, M06-2X ${ }^{102}$ and B3LYP, ${ }^{103,104}$ were used in the DFT calculations. The B3LYP calculation was augmented by Grimme's D3 dispersion with Becke-Johnson damping function (B3LYP-D3). ${ }^{105,106}$ The crystal structure of this system is taken as the initial geometry for all geometry optimizations. The optimized structures are shown in Figure 5.

The optimized structures obtained with three different methods are quite similar to the X-ray crystal structure. The RMSDs of bond lengths (bonds between nonhydrogen atoms) between the structures optimized by the three methods (CIM-MP2, M06-2X, and B3LYP-D3) and the crystal structure are $0.030 \AA, 0.029 \AA$, and $0.028 \AA$, respectively. The RMSDs of the bond lengths between the CIM-MP2 optimized structure and the DFT optimized structures are $0.0047 \AA$ for M06-2X and $0.0043 \AA$ for B3LYP-D3, respectively. All these structures have nine intramolecular hydrogen bonds to maintain a 310 -helix structure. For a better comparison, the $\mathrm{N} \cdots \mathrm{O}$ and $\mathrm{H} \cdots \mathrm{O}$ bond lengths calculated with three methods are listed in Table 6. The MADs of the $\mathrm{N} \cdots \mathrm{O}$ bond lengths of the three optimized structures with respect to those of the crystal structure are $0.08 \AA$ (CIM-MP2), $0.06 \AA$ (M06-2X), and $0.07 \AA$ (B3LYP-D3), respectively. The $\mathrm{H} \cdots \mathrm{O}$ bond lengths of the CIM-MP2 method are also similar to those of the two DFT methods. It should be noted that the CIM-MP2 optimized structure may be further improved if a triple- $\zeta$ basis set is employed. All these results demostrate that CIM-MP2 provides a satisfactory description for this oligopeptide. 


\section{SUMMARY AND FUTURE PERSPECTIVES}

We have presented an efficient implementation of the CIM-MP2 gradient. In our approach, we proposed a single-cluster method to evaluate the gradient contributions from the nonseparable term of the two-body density matrix. The evaluation of other terms in the CIM-MP2 energy gradient is done by constructing the density matrices of the whole system with the contributions from all clusters. By analyzing the errors from test calculations, we find that tightened thresholds should be used if the basis set contains diffuse functions. Benchmark calculations show that with suitable thresholds the present CIM-MP2 gradient algorithm can accurately reproduce the conventional MP2 gradients. With the CIM-MP2 gradients, we have obtained optimized structures for three medium-sized molecules. A comparison of the CIM-MP2 optimized structures with the corresponding MP2 optimized structures indicates that the CIM-MP2 method can reproduce the conventional MP2 geometries. For an oligopeptide with 174 atoms, we have optimized its structure with the CIM-MP2 method level at the cc-pVDZ basis set. The optimized structural parameters are in good agreement with the X-ray crystal structure and the DFT-optimized geometries (with M06-2X and B3LYP-D3 functionals). These results show that the present CIM-MP2 gradient can be used for efficient and accurate geometry optimization of large systems, especially for those systems where DFT is not accurate enough.

The CIM-MP2 method is naturally parallelized, due to independent calculations of various clusters. Its performance can be further improved by parallelizing the calculation for each cluster and employing density fitting approximation for the AO integrals. Development along these two directions is underway in our laboratory.

\section{Supplementary Material}

Refer to Web version on PubMed Central for supplementary material.

\section{ACKNOWLEDGMENTS}

The authors are grateful to Prof. Wolinski for his help with MP2 gradient program in PQS package. Z. Ni is indebted to Mr. Jingxiang Zou for valuable discussions.

Funding

This work was supported primarily by the National Natural Science Foundation of China (Grant Nos. 21833002, 21673110, and 21873046). It was also supported by the U.S. National Science Foundation (Grant Nos. CHE-1213870 and DMR-1609650), by the U.S. National Institute of Health under Grant No. 1R01GM120578, and by the Arkansas Biosciences Institute, the major research component of the Arkansas Tobacco Settlement Proceeds Act of 2000. Most of the calculations in this work were done at the High Performance Computing Center of Nanjing University, and some calculations were done at the Arkansas High Performance Computer Center.

\section{REFERENCES}

(1). Pulay P Localizability of dynamic electron correlation. Chem. Phys. Lett 1983, 100, 151-154.

(2). Sæbø S; Pulay P Local configuration interaction: An efficient approach for larger molecules. Chem. Phys. Lett 1985, 113, 13-18.

(3). Förner W; Ladik J; Otto P; Čížek J Coupled-cluster studies. II. The role of localization in correlation calculations on extended systems. Chem. Phys 1985, 97, 251-262. 
(4). Saeb $\emptyset$ S; Pulay P Fourth-order Møller-Plessett perturbation theory in the local correlation treatment. I. Method. J. Chem. Phys 1987, 86, 914-922.

(5). Saebø S; Pulay P The local correlation treatment. II. Implementation and tests. J. Chem. Phys 1988, 88, 1884-1890.

(6). Fórner W; Č́̌žek J; Otto P; Ladik J; Steinborn EO Coupled-cluster studies. I. Application to small molecules, basis set dependences. Chem. Phys 1985, 97, 235-249.

(7). Pulay P; Saebø S Orbital-invariant formulation and second-order gradient evaluation in MøllerPlesset perturbation theory. Theor. Chim. Acta 1986, 69, 357-368.

(8). Hampel C; Werner HJ Local treatment of electron correlation in coupled cluster theory. J. Chem. Phys 1996, 104, 6286-6297.

(9). Maslen PE; Head-Gordon M Non-iterative local second order Møller-Plesset theory. Chem. Phys. Lett 1998, 283, 102-108.

(10). Maslen PE; Head-Gordon M Noniterative local second order Møller-Plesset theory: Convergence with local correlation space. J. Chem. Phys 1998, 109, 7093-7099.

(11). Ayala PY; Scuseria GE Linear scaling second-order Møller-Plesset theory in the atomic orbital basis for large molecular systems. J. Chem. Phys 1999, 110, 3660-3671.

(12). Schütz M; Hetzer G; Werner H-J Low-order scaling local electron correlation methods. I. Linear scaling local MP2. J. Chem. Phys 1999, 111, 5691-5705.

(13). Scuseria GE; Ayala PY Linear scaling coupled cluster and perturbation theories in the atomic orbital basis. J. Chem. Phys 1999, 111, 8330-8343.

(14). Schütz M Low-order scaling local electron correlation methods. III. Linear scaling local perturbative triples correction (T). J. Chem. Phys 2000, 113, 9986-10001.

(15). Schütz M; Werner H-J Local perturbative triples correction (T) with linear cost scaling. Chem. Phys. Lett 2000, 318, 370-378.

(16). Schütz M; Werner H-J Low-order scaling local electron correlation methods. IV. Linear scaling local coupled-cluster (LCCSD). J. Chem. Phys 2001, 114, 661-681.

(17). Schutz M A new, fast, semi-direct implementation of linear scaling local coupled cluster theory. Phys. Chem. Chem. Phys 2002, 4, 3941-3947.

(18). Schütz M Low-order scaling local electron correlation methods. V. Connected triples beyond (T): Linear scaling local CCSDT-1b. J. Chem. Phys 2002, 116, 8772-8785.

(19). Neese F; Hansen A; Liakos DG Efficient and accurate approximations to the local coupled cluster singles doubles method using a truncated pair natural orbital basis. J. Chem. Phys 2009, 131, 064103. [PubMed: 19691374]

(20). Neese F; Wennmohs F; Hansen A Efficient and accurate local approximations to coupledelectron pair approaches: An attempt to revive the pair natural orbital method. J. Chem. Phys 2009, 130, 114108. [PubMed: 19317532]

(21). Werner H-J; Schütz M An efficient local coupled cluster method for accurate thermochemistry of large systems. J. Chem. Phys 2011, 135, 144116. [PubMed: 22010707]

(22). Yang J; Kurashige Y; Manby FR; Chan GKL Tensor factorizations of local second-order MøllerPlesset theory. J. Chem. Phys 2011, 134, 044123. [PubMed: 21280703]

(23). Kurashige Y; Yang J; Chan GK-L; Manby FR Optimization of orbital-specific virtuals in local Møller-Plesset perturbation theory. J. Chem. Phys 2012, 136, 124106. [PubMed: 22462834]

(24). Riplinger C; Neese F An efficient and near linear scaling pair natural orbital based local coupled cluster method. J. Chem. Phys 2013, 138, 034106. [PubMed: 23343267]

(25). Riplinger C; Sandhoefer B; Hansen A; Neese F Natural triple excitations in local coupled cluster calculations with pair natural orbitals. J. Chem. Phys 2013, 139, 134101. [PubMed: 24116546]

(26). Schütz M; Yang J; Chan GK-L; Manby FR; Werner H-J The orbital-specific virtual local triples correction: OSV-L(T). J. Chem. Phys 2013, 138, 054109. [PubMed: 23406100]

(27). Schwilk M; Usvyat D; Werner H-J Communication: Improved pair approximations in local coupled-cluster methods. J. Chem. Phys 2015, 142, 121102. [PubMed: 25833558]

(28). Werner H-J; Knizia G; Krause C; Schwilk M; Dornbach M Scalable Electron Correlation Methods I.: PNO-LMP2 with Linear Scaling in the Molecular Size and Near-Inverse-Linear 
Scaling in the Number of Processors. J. Chem. Theory Comput 2015, 11, 484-507. [PubMed: 26580908]

(29). Riplinger C; Pinski P; Becker U; Valeev EF; Neese F Sparse maps-A systematic infrastructure for reduced-scaling electronic structure methods. II. Linear scaling domain based pair natural orbital coupled cluster theory. J. Chem. Phys 2016, 144, 024109. [PubMed: 26772556]

(30). Schwilk M; Ma Q; Köppl C; Werner H-J Scalable Electron Correlation Methods. 3. Efficient and Accurate Parallel Local Coupled Cluster with Pair Natural Orbitals (PNO-LCCSD). J. Chem. Theory Comput 2017, 13, 3650-3675. [PubMed: 28661673]

(31). Guo Y; Riplinger C; Becker U; Liakos DG; Minenkov Y; Cavallo L; Neese F Communication: An improved linear scaling perturbative triples correction for the domain based local pair-natural orbital based singles and doubles coupled cluster method [DLPNO-CCSD(T)]. J. Chem. Phys 2018, 148, 011101. [PubMed: 29306283]

(32). Head-Gordon M; Maslen PE; White CA A tensor formulation of many-electron theory in a nonorthogonal single-particle basis. J. Chem. Phys 1998, 108, 616-625.

(33). Kats D; Korona T; Schütz M Local CC2 electronic excitation energies for large molecules with density fitting. J. Chem. Phys 2006, 125, 104106. [PubMed: 16999514]

(34). Yang J; Chan GK-L; Manby FR; Schütz M; Werner H-J The orbital-specific-virtual local coupled cluster singles and doubles method. J. Chem. Phys 2012, 136, 144105. [PubMed: 22502499]

(35). Liakos DG; Neese F Is It Possible To Obtain Coupled Cluster Quality Energies at near Density Functional Theory Cost? Domain-Based Local Pair Natural Orbital Coupled Cluster vs Modern Density Functional Theory. J. Chem. Theory Comput 2015, 11, 4054-4063. [PubMed: 26575901]

(36). Liakos DG; Sparta M; Kesharwani MK; Martin JML; Neese F Exploring the Accuracy Limits of Local Pair Natural Orbital Coupled-Cluster Theory. J. Chem. Theory Comput 2015, 11, 15251539. [PubMed: 26889511]

(37). Pinski P; Riplinger C; Valeev EF; Neese F Sparse maps-A systematic infrastructure for reduced-scaling electronic structure methods. I. An efficient and simple linear scaling local MP2 method that uses an intermediate basis of pair natural orbitals. J. Chem. Phys 2015, 143, 034108. [PubMed: 26203015]

(38). Förner W Coupled cluster studies. IV. Analysis of the correlated wavefunction in canonical and localized orbital basis for ethylene, carbon monoxide, and carbon dioxide. Chem. Phys 1987, 114, 21-35.

(39). Stoll H Correlation energy of diamond. Phys. Rev. B: Condens. Matter Mater. Phys 1992, 46, 6700-6704.

(40). Stoll H The correlation energy of crystalline silicon. Chem. Phys. Lett 1992, 191, 548-552.

(41). Rościszewski K; Doll K; Paulus B; Fulde P; Stoll H Ground-state properties of rutile: Electroncorrelation effects. Phys. Rev. B: Condens. Matter Mater. Phys 1998, 57, 14667-14672.

(42). Flocke N; Bartlett RJ A natural linear scaling coupled-cluster method. J. Chem. Phys 2004, 121, 10935-10944. [PubMed: 15634043]

(43). Stoll H; Paulus B; Fulde P On the accuracy of correlation-energy expansions in terms of local increments. J. Chem. Phys 2005, 123, 144108. [PubMed: 16238375]

(44). Friedrich J; Hanrath M; Dolg M Fully automated implementation of the incremental scheme: Application to CCSD energies for hydrocarbons and transition metal compounds. J. Chem. Phys 2007, 126, 154110. [PubMed: 17461617]

(45). Friedrich J; Dolg M Fully Automated Incremental Evaluation of MP2 and CCSD(T) Energies: Application to Water Clusters. J. Chem. Theory Comput 2009, 5, 287-294. [PubMed: 26610105]

(46). Li S; Ma J; Jiang Y Linear scaling local correlation approach for solving the coupled cluster equations of large systems. J. Comput. Chem 2002, 23, 237-244. [PubMed: 11924736]

(47). Li S; Shen J; Li W; Jiang Y An efficient implementation of the "cluster-in-molecule" approach for local electron correlation calculations. J. Chem. Phys 2006, 125, 074109. [PubMed: 16942324]

(48). Li W; Piecuch P; Gour JR; Li S Local correlation calculations using standard and renormalized coupled-cluster approaches. J. Chem. Phys 2009, 131, 114109. [PubMed: 19778102] 
(49). Li W; Piecuch P Multilevel Extension of the Cluster-in-Molecule Local Correlation Methodology: Merging Coupled-Cluster and Møller-Plesset Perturbation Theories. J. Phys. Chem. A 2010, 114, 6721-6727. [PubMed: 20496942]

(50). Li W; Piecuch P Improved Design of Orbital Domains within the Cluster-in-Molecule Local Correlation Framework: Single-Environment Cluster-in-Molecule Ansatz and Its Application to Local Coupled-Cluster Approach with Singles and Doubles. J. Phys. Chem. A 2010, 114, 86448657. [PubMed: 20373794]

(51). Li W; Guo Y; Li S A refined cluster-in-molecule local correlation approach for predicting the relative energies of large systems. Phys. Chem. Chem. Phys 2012, 14, 7854-7862. [PubMed: 22456726]

(52). Guo Y; Li W; Li S Improved Cluster-in-Molecule Local Correlation Approach for Electron Correlation Calculation of Large Systems. J. Phys. Chem. A 2014, 118, 8996-9004. [PubMed: 24963784]

(53). Guo Y; Li W; Yuan D; Li S The relative energies of polypeptide conformers predicted by linear scaling second-order Møller-Plesset perturbation theory. Sci. China: Chem 2014, 57, 1393-1398.

(54). Li W; Li S Cluster-in-molecule local correlation method for large systems. Sci. China: Chem 2014, 57, 78-86.

(55). Li W; Ni Z; Li S Cluster-in-molecule local correlation method for post-Hartree-Fock calculations of large systems. Mol. Phys 2016, 114, 1447-1460.

(56). Ni Z; Li W; Li S Fully optimized implementation of the cluster-in-molecule local correlation approach for electron correlation calculations of large systems. J. Comput. Chem 2019, 40, 1130-1140. [PubMed: 30362560]

(57). Ziółkowski M; Jansík B; Kjærgaard T; Jørgensen P Linear scaling coupled cluster method with correlation energy based error control. J. Chem. Phys 2010, 133, 014107. [PubMed: 20614959]

(58). Kristensen K; Hoyvik I-M; Jansik B; Jorgensen P; Kjaergaard T; Reine S; Jakowski J MP2 energy and density for large molecular systems with internal error control using the DivideExpand-Consolidate scheme. Phys. Chem. Chem. Phys 2012, 14, 15706-15714. [PubMed: 23090588]

(59). Kjærgaard $\mathrm{T}$ The Laplace transformed divide-expand-consolidate resolution of the identity second-order Møller-Plesset perturbation (DEC-LT-RIMP2) theory method. J. Chem. Phys 2017, 146, 044103. [PubMed: 28147513]

(60). Kjaergaard T; Baudin P; Bykov D; Kristensen K; Jorgensen P The divide-expand-consolidate coupled cluster scheme. WIREs. Comput. Mol. Sci 2017, 7, No. e1319.

(61). Boughton JW; Pulay P Comparison of the boys and Pipek-Mezey localizations in the local correlation approach and automatic virtual basis selection. J. Comput. Chem 1993, 14, 736-740.

(62). Feyereisen M; Fitzgerald G; Komornicki A Use of approximate integrals in ab initio theory. An application in MP2 energy calculations. Chem. Phys. Lett 1993, 208, 359-363.

(63). Piecuch P; Włoch M Renormalized coupled-cluster methods exploiting left eigenstates of the similarity-transformed Hamiltonian. J. Chem. Phys 2005, 123, 224105. [PubMed: 16375468]

(64). Rolik Z; Kállay M A general-order local coupled-cluster method based on the cluster-in-molecule approach. J. Chem. Phys 2011, 135, 104111. [PubMed: 21932880]

(65). Rolik Z; Szegedy L; Ladjánszki I; Ladóczki B; Kállay M An efficient linear-scaling CCSD(T) method based on local natural orbitals. J. Chem. Phys 2013, 139, 094105. [PubMed: 24028100]

(66). Nagy PR; Samu G; Kállay M An Integral-Direct Linear-Scaling Second-Order Møller-Plesset Approach. J. Chem. Theory Comput 2016, 12, 4897-4914. [PubMed: 27618512]

(67). Nagy PR; Samu G; Kállay M Optimization of the Linear-Scaling Local Natural Orbital CCSD(T) Method: Improved Algorithm and Benchmark Applications. J. Chem. Theory Comput 2018, 14, 4193-4215. [PubMed: 29965753]

(68). Guo Y; Becker U; Neese F Comparison and combination of "direct" and fragment based local correlation methods: Cluster in molecules and domain based local pair natural orbital perturbation and coupled cluster theories. J. Chem. Phys 2018, 148, 124117. [PubMed: 29604807]

(69). Pople JA; Krishnan R; Schlegel HB; Binkley JS Derivative Studies in Hartree-Fock and MøllerPlesset Theories. Int. J. Quantum Chem 1979, 16, 225-241. 
(70). Jørgensen P; Helgaker T Møller-Plesset energy derivatives. J. Chem. Phys 1988, 89, 1560-1570.

(71). Helgaker T; Jørgensen P; Handy NC A numerically stable procedure for calculating MøllerPlesset energy derivatives, derived using the theory of Lagrangians. Theor. Chim. Acta 1989, 76, 227-245.

(72). Frisch MJ; Head-Gordon M; Pople JA A direct MP2 gradient method. Chem. Phys. Lett 1990, $166,275-280$.

(73). Frisch MJ; Head-Gordon M; Pople JA Semi-direct algorithms for the MP2 energy and gradient. Chem. Phys. Lett 1990, 166, 281-289.

(74). Haase F; Ahlrichs R Semidirect MP2 gradient evaluation on workstation computers: The MPGRAD program. J. Comput. Chem 1993, 14, 907-912.

(75). Nielsen IMB A new direct MP2 gradient algorithm with implementation on a massively parallel computer. Chem. Phys. Lett 1996, 255, 210-216.

(76). Fletcher BGD; Rendell AP; Sherwood P A parallel second-order Møller-Plesset gradient. Mol. Phys 1997, 91, 431-438.

(77). Head-Gordon M An improved semidirect MP2 gradient method. Mol. Phys 1999, 96, 673-679.

(78). Aikens CM; Webb SP; Bell RL; Fletcher GD; Schmidt MW; Gordon MS A derivation of the frozen-orbital unrestricted open-shell and restricted closed-shell second-order perturbation theory analytic gradient expressions. Theor. Chem. Acc 2003, 110, 233-253.

(79). Saebø S; Baker J; Wolinski K; Pulay P An efficient atomic orbital based second-order MøllerPlesset gradient program. J. Chem. Phys 2004, 120, 11423-11431. [PubMed: 15268176]

(80). Ishimura K; Pulay P; Nagase S New parallel algorithm for MP2 energy gradient calculations. J. Comput. Chem 2007, 28, 2034-2042. [PubMed: 17450568]

(81). El Azhary A; Rauhut G; Pulay P; Werner H-J Analytical energy gradients for local second-order Møller-Plesset perturbation theory. J. Chem. Phys 1998, 108, 5185-5193.

(82). Schütz M; Werner H-J; Lindh R; Manby FR Analytical energy gradients for local second-order Møller-Plesset perturbation theory using density fitting approximations. J. Chem. Phys 2004, 121, 737-750. [PubMed: 15260600]

(83). Dornbach M; Werner H-J Analytical energy gradients for local second-order Møller-Plesset perturbation theory using intrinsic bond orbitals. Mol. Phys 2019, 117, 1252.

(84). Frank MS; Schmitz G; Hättig C The PNO-MP2 gradient and its application to molecular geometry optimizations. Mol. Phys 2017, 115, 343-356.

(85). Pinski P; Neese F Communication: Exact analytical derivatives for the domain-based local pair natural orbital MP2 method (DLPNO-MP2). J. Chem. Phys 2018, 148, 031101. [PubMed: 29352787]

(86). Kristensen K; Jørgensen P; Jansík B; Kjærgaard T; Reine S Molecular gradient for second-order Møller-Plesset perturbation theory using the divide-expand-consolidate (DEC) scheme. J. Chem. Phys 2012, 137, 114102. [PubMed: 22998244]

(87). Bykov D; Kristensen K; Kjærgaard T The molecular gradient using the divide-expandconsolidate resolution of the identity second-order Møller-Plesset perturbation theory: The DECRI-MP2 gradient. J. Chem. Phys 2016, 145, 024106. [PubMed: 27421396]

(88). Fedorov DG; Kitaura K Second order Møller-Plesset perturbation theory based upon the fragment molecular orbital method. J. Chem. Phys 2004, 121, 2483-2490. [PubMed: 15281845]

(89). Nagata T; Fedorov DG; Ishimura K; Kitaura K Analytic energy gradient for second-order MøllerPlesset perturbation theory based on the fragment molecular orbital method. J. Chem. Phys 2011, 135, 044110. [PubMed: 21806093]

(90). Deev V; Collins MA Approximate ab initio energies by systematic molecular fragmentation. J. Chem. Phys 2005, 122, 154102. [PubMed: 15945620]

(91). Ganesh V; Dongare RK; Balanarayan P; Gadre SR Molecular tailoring approach for geometry optimization of large molecules: Energy evaluation and parallelization strategies. J. Chem. Phys 2006, 125, 104109. [PubMed: 16999517]

(92). Hua W; Fang T; Li W; Yu J-G; Li S Geometry Optimizations and Vibrational Spectra of Large Molecules from a Generalized Energy-Based Fragmentation Approach. J. Phys. Chem. A 2008, 112, 10864-10872. [PubMed: 18837491] 
(93). Kobayashi M; Nakai H An effective energy gradient expression for divide-and-conquer secondorder Møller-Plesset perturbation theory. J. Chem. Phys 2013, 138, 044102. [PubMed: 23387563]

(94). Pulay P; Saebø S; Meyer W An efficient reformulation of the closed-shell self-consistent electron pair theory. J. Chem. Phys 1984, 81, 1901-1905.

(95). Handy NC; Schaefer HF On the evaluation of analytic energy derivatives for correlated wave functions. J. Chem. Phys 1984, 81, 5031-5033.

(96). Boys SF Construction of Some Molecular Orbitals to Be Approximately Invariant for Changes from One Molecule to Another. Rev. Mod. Phys 1960, 32, 296-299.

(97). Foster JM; Boys SF Canonical Configurational Interaction Procedure. Rev. Mod. Phys 1960, 32, 300-302.

(98). Pipek J; Mezey PG A fast intrinsic localization procedure applicable for ab initio and semiempirical linear combination of atomic orbital wave functions. J. Chem. Phys 1989, 90, 4916-4926.

(99). Baker J; Wolinski K; Janowski T; Saebø S; Pulay P PQS version 4.1; Parallel Quantum Solutions LLC: Fayetteville, AR, U.S.A., 2011.

(100). Frisch MJ; Trucks GW; Schlegel HB; Scuseria GE; Robb MA; Cheeseman JR; Scalmani G; Barone V; Petersson GA; Nakatsuji H; Li X; Caricato M; Marenich AV; Bloino J; Janesko BG; Gomperts R; Mennucci B; Hratchian HP; Ortiz JV; Izmaylov AF; Sonnenberg JL; Williams; Ding F; Lipparini F; Egidi F; Goings J; Peng B; Petrone A; Henderson T; Ranasinghe D; Zakrzewski VG; Gao J; Rega N; Zheng G; Liang W; Hada M; Ehara M; Toyota K; Fukuda R; Hasegawa J; Ishida M; Nakajima T; Honda Y; Kitao O; Nakai H; Vreven T; Throssell K; Montgomery JA Jr; Peralta JE; Ogliaro F; Bearpark MJ; Heyd JJ; Brothers EN; Kudin KN; Staroverov VN; Keith TA; Kobayashi R; Normand J; Raghavachari K; Rendell AP; Burant JC; Iyengar SS; Tomasi J; Cossi M; Millam JM; Klene M; Adamo C; Cammi R; Ochterski JW; Martin RL; Morokuma K; Farkas O; Foresman JB; Fox DJ Gaussian 16, Revision A.03; Gaussian Inc.: Wallingford, CT, 2016.

(101). Gessmann R; Brückner H; Petratos K Three complete turns of a 310-helix at atomic resolution: the crystal structure of Z-(Aib)11-OtBu. J. Pept. Sci 2003, 9, 753-762. [PubMed: 14658794]

(102). Zhao Y; Truhlar DG The M06 suite of density functionals for main group thermochemistry, thermochemical kinetics, non-covalent interactions, excited states, and transition elements: two new functionals and systematic testing of four M06-class functionals and 12 other functionals. Theor. Chem. Acc 2008, 120, 215-241.

(103). Becke AD Density-functional thermochemistry. III. The role of exact exchange. J. Chem. Phys 1993, 98, 5648-5652.

(104). Lee C; Yang W; Parr RG Development of the Colle-Salvetti correlation-energy formula into a functional of the electron density. Phys. Rev. B: Condens. Matter Mater. Phys 1988, 37, 785-789.

(105). Grimme S; Antony J; Ehrlich S; Krieg H A consistent and accurate ab initio parametrization of density functional dispersion correction (DFT-D) for the 94 elements H-Pu. J. Chem. Phys 2010, 132, 154104. [PubMed: 20423165]

(106). Grimme S; Ehrlich S; Goerigk L Effect of the damping function in dispersion corrected density functional theory. J. Comput. Chem 2011, 32, 1456-1465. [PubMed: 21370243] 


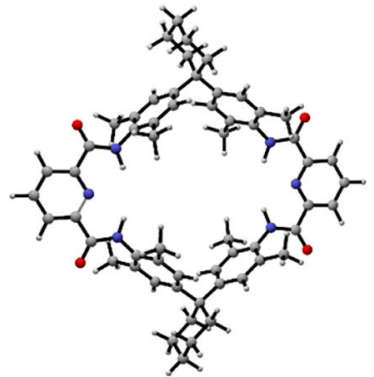

(1) $\mathrm{C}_{58} \mathrm{H}_{62} \mathrm{~N}_{6} \mathrm{O}_{4}$

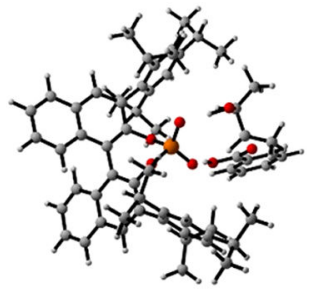

(4) $\mathrm{C}_{61} \mathrm{H}_{71} \mathrm{O}_{7} \mathrm{P}$

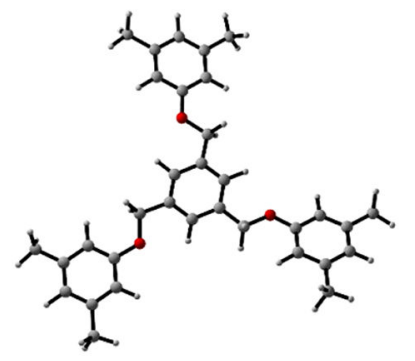

(2) $\mathrm{C}_{33} \mathrm{H}_{36} \mathrm{O}_{3}$

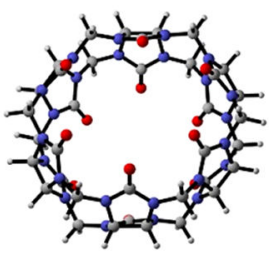

(5) $\mathrm{C}_{36} \mathrm{H}_{36} \mathrm{~N}_{24} \mathrm{O}_{12}$

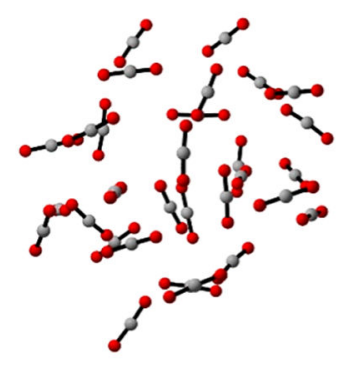

(3) $\left(\mathrm{CO}_{2}\right)_{30}$

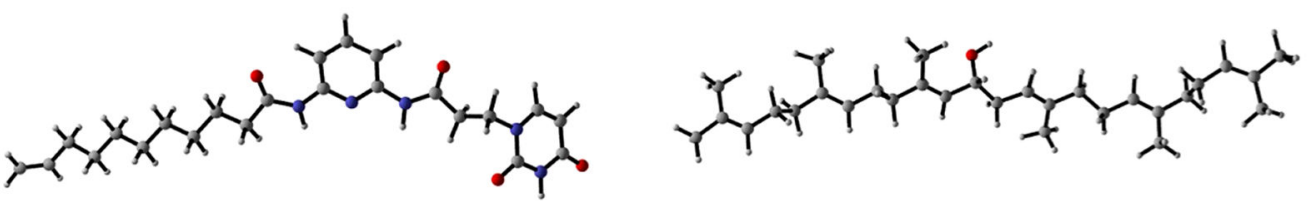

(7) $\mathrm{C}_{23} \mathrm{H}_{31} \mathrm{~N}_{5} \mathrm{O}_{4}$

(8) $\mathrm{C}_{30} \mathrm{H}_{50} \mathrm{O}$

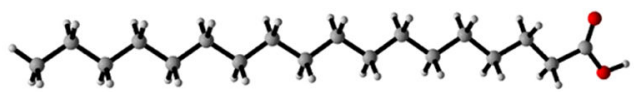

(9) $\mathrm{C}_{18} \mathrm{H}_{36} \mathrm{O}_{2}$

Figure 1.

Structures of the molecules used in the benchmark calculations.

J Chem Theory Comput. Author manuscript; available in PMC 2020 June 11. 


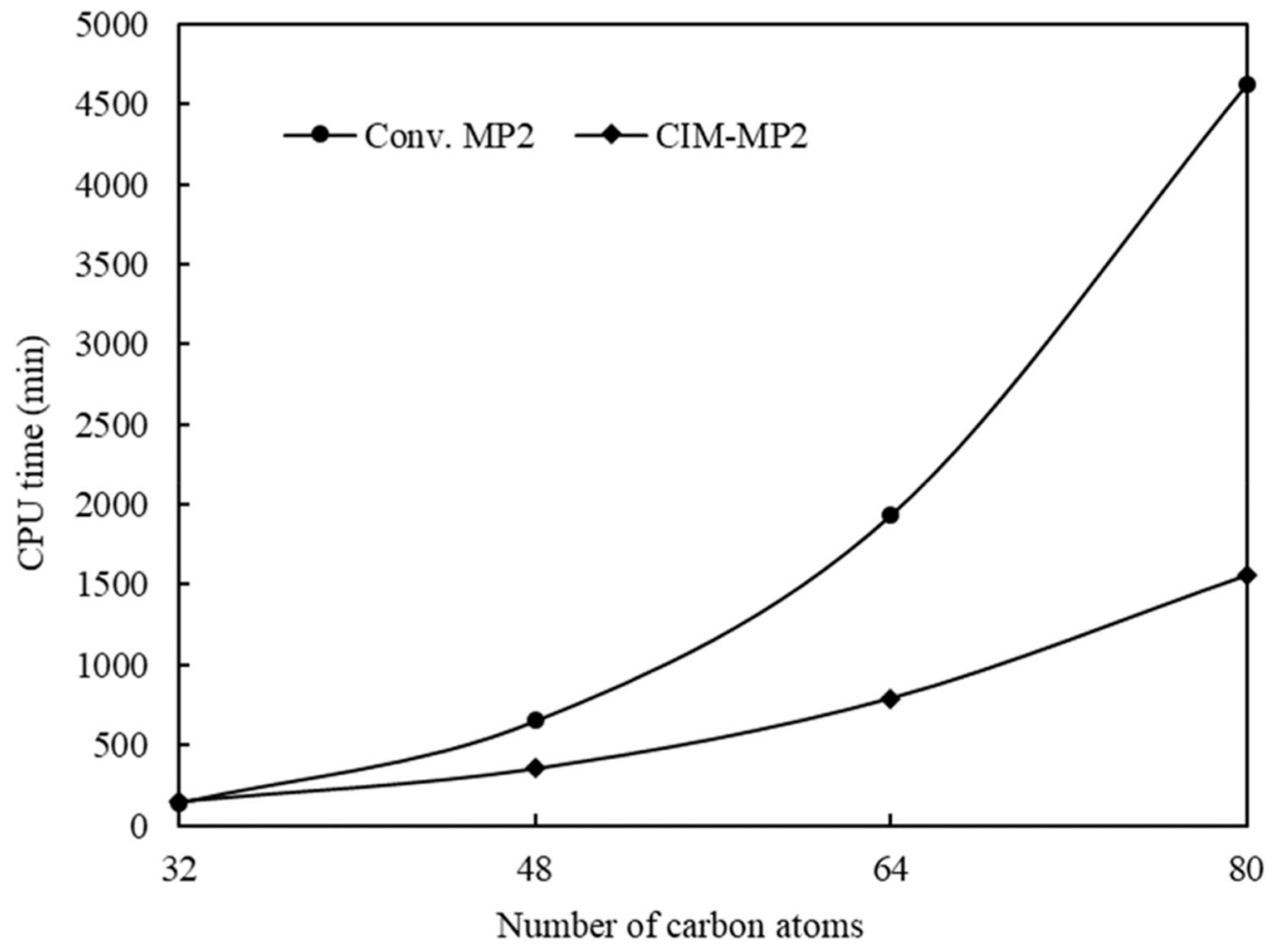

Figure 2.

CPU times (in min) for CIM-MP2 and conventional MP2 gradients of a series of hydrocarbons with different numbers of carbon atoms. 


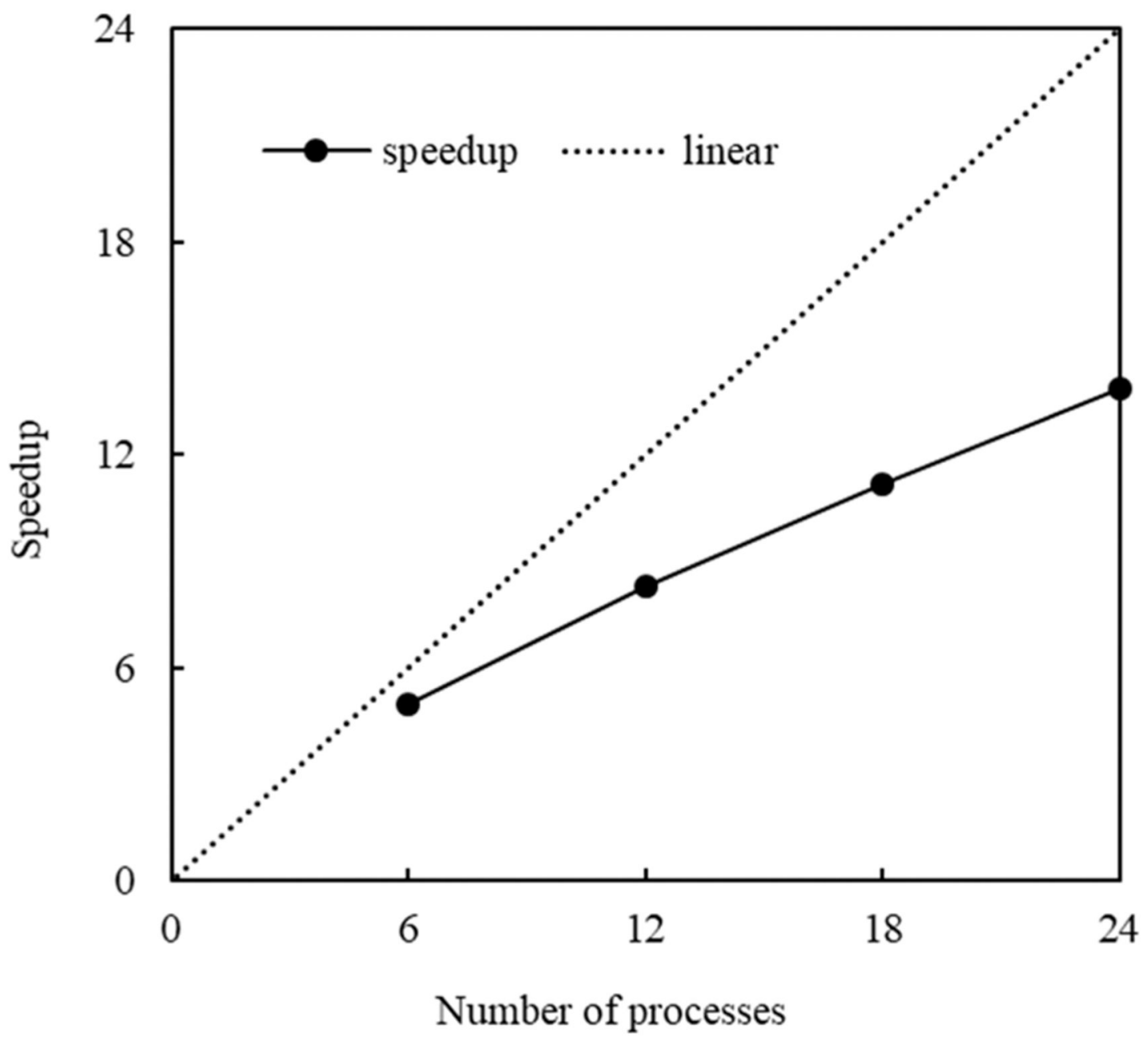

Figure 3.

Speedups on different numbers of CPU cores. 

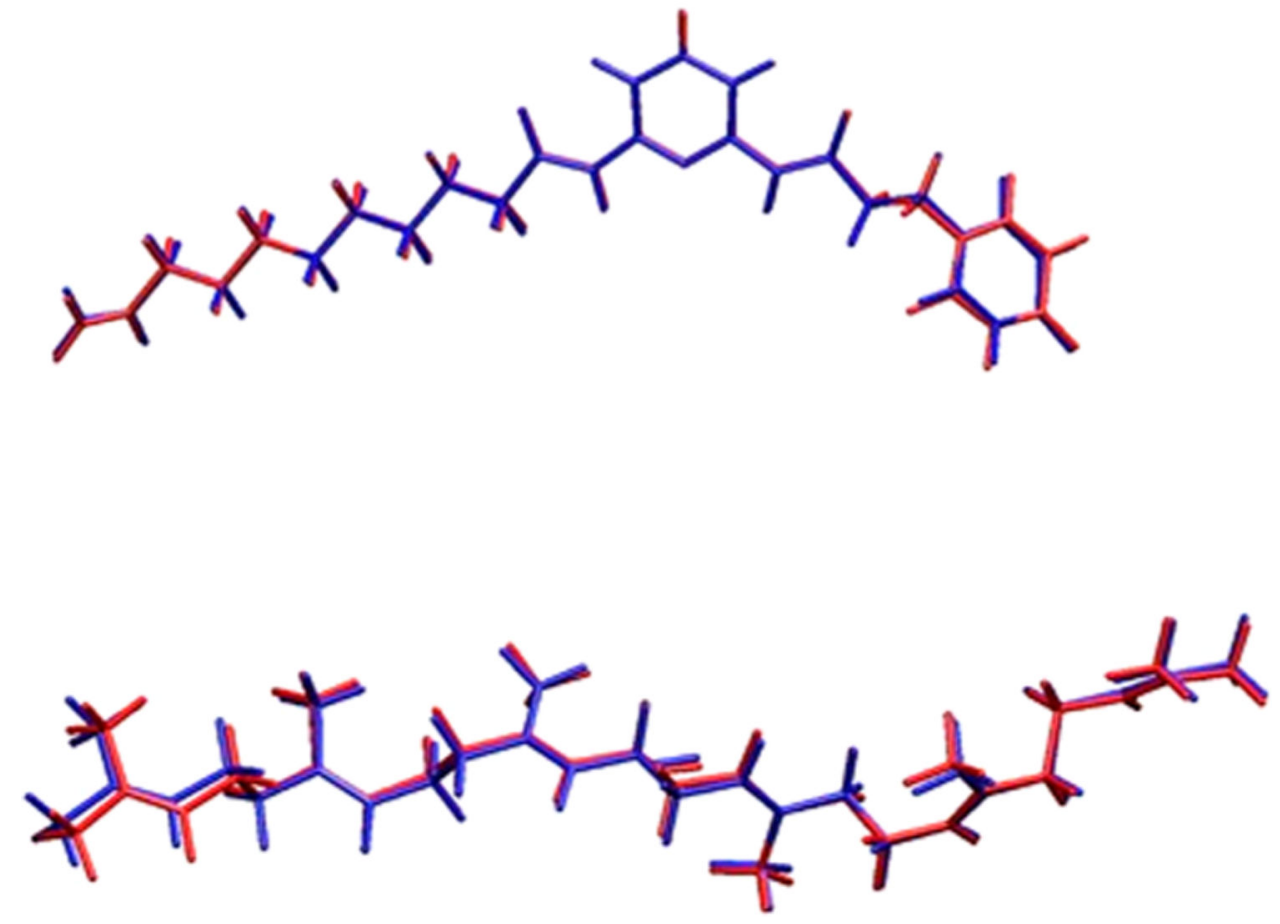

Figure 4.

Superposition between the optimized structures of the two systems (7) and (8) (in Figure 1) obtained with the CIM-MP2 (red) and conventional MP2 (blue) methods. 


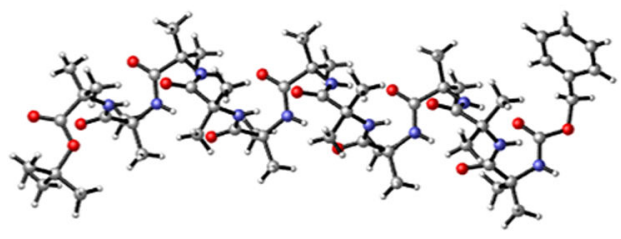

crystal

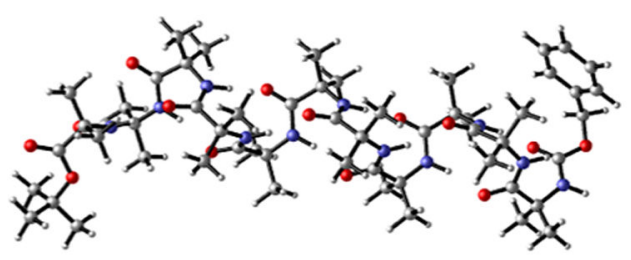

M06-2X

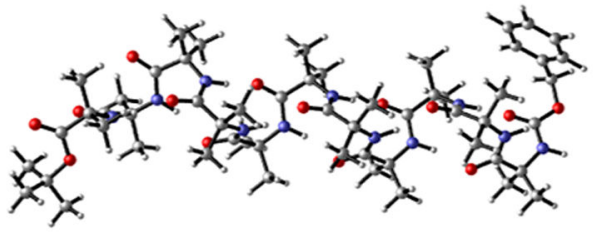

CIM-MP2

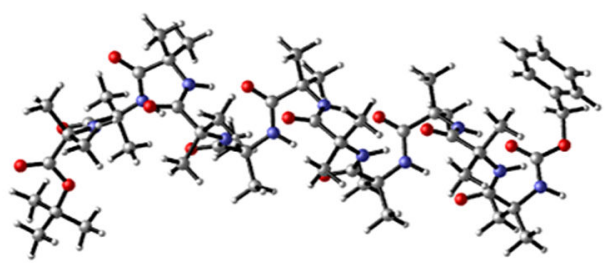

B3LYP-D3

Figure 5.

Comparison of the crystal structure and optimized structures obtained with CIM-MP2, M06-2X, and B3LYP-D3 for an oligopeptide. 
Table 1.

Maximum Deviation (in $\mathrm{m} E_{\mathrm{h}} / \mathrm{a}_{0}$ ) of the Different Terms in $3 N(N$ is the number of atoms) Cartesian Gradient Components Calculated with the CIM-MP2 Approach (with two basis sets and different parameters) Relative to the Conventional MP2 Result

\begin{tabular}{|c|c|c|c|c|c|}
\hline \multirow[b]{2}{*}{ terms } & \multicolumn{2}{|c|}{ 6-31G(d) } & \multicolumn{2}{|c|}{$6-31+G$} & \multirow[b]{2}{*}{$10.0 / 0.0$} \\
\hline & $5.5 / 0.05$ & 8.0/0.01 & $5.5 / 0.05$ & $8.0 / 0.01$ & \\
\hline $\mathbf{X}^{\prime}$ & 0.04 & -0.11 & 39.95 & 2.62 & 0.92 \\
\hline $\mathbf{W}^{\prime}$ & 0.14 & 0.26 & -39.3 & -2.63 & -0.96 \\
\hline$\Gamma^{\mathrm{NS}}$ & 0.22 & -0.02 & 103.67 & 51.89 & 5.75 \\
\hline total & 0.40 & 0.13 & 104.32 & 51.88 & 5.71 \\
\hline
\end{tabular}


Table 2.

Deviation (in $\mathrm{m} E_{\mathrm{h}} / \mathrm{a}_{0}$ ) of the $x$-Components of the Gradient Contributions on the First $10 \mathrm{C}$ Atoms from the NS Term Calculated with Two Different Methods (with respect to the results from the conventional method) ${ }^{a}$

\begin{tabular}{ccc}
\hline C atom & cluster-averaged method & single-cluster method \\
1 & -2.25 & 0.04 \\
2 & -0.25 & 0.09 \\
3 & 0.54 & 0.00 \\
4 & 0.33 & -0.02 \\
5 & 16.48 & 0.17 \\
6 & 83.31 & -0.09 \\
7 & -83.16 & 0.23 \\
8 & -97.90 & -0.78 \\
9 & 72.59 & 0.51 \\
10 & -74.27 & 1.05 \\
\hline
\end{tabular}

${ }^{a}$ The 6-31+G basis set is used, and 8.0/0.01 parameters are used for CIM-MP2 calculations. 
Table 3.

Comparison of the CIM-MP2 Gradients with the Conventional MP2 Gradients (in $\mathrm{m} E_{\mathrm{h}} / \mathrm{a}_{0}$ ) for Nine Systems at Various Basis Sets ${ }^{a}$

\begin{tabular}{cclccc}
\hline system & chemical formula & basis set & $N_{\text {bas }}$ & MAXD & MAD \\
$(1)$ & $\mathrm{C}_{58} \mathrm{H}_{62} \mathrm{~N}_{6} \mathrm{O}_{4}$ & def2-SVP & 1076 & 0.75 & 0.14 \\
$(2)$ & $\mathrm{C}_{33} \mathrm{H}_{36} \mathrm{O}_{3}$ & def2-TZVP & 1332 & 2.69 & 0.23 \\
$(3)$ & $\left(\mathrm{CO}_{2}\right)_{30}$ & cc-pVDZ & 1260 & 2.78 & 0.44 \\
$(4)$ & $\mathrm{C}_{61} \mathrm{H}_{71} \mathrm{O}_{7} \mathrm{P}$ & $6-311 \mathrm{G}(\mathrm{d}, \mathrm{p})$ & 1325 & 1.30 & 0.13 \\
$(5)$ & $\mathrm{C}_{36} \mathrm{H}_{36} \mathrm{~N}_{24} \mathrm{O}_{12}$ & $6-311 \mathrm{G}(\mathrm{d}, \mathrm{p})$ & 1512 & 0.71 & 0.12 \\
$(6)$ & $\mathrm{C}_{24} \mathrm{H}_{42} \mathrm{~N}_{8} \mathrm{O}_{10}$ & $6-31+\mathrm{G}$ & 630 & 0.67 & 0.05 \\
$(7)$ & $\mathrm{C}_{23} \mathrm{H}_{31} \mathrm{~N}_{5} \mathrm{O}_{4}$ & $6-31+\mathrm{G}$ & 478 & 3.30 & 0.21 \\
$(8)$ & $\mathrm{C}_{30} \mathrm{H}_{50} \mathrm{O}$ & $6-31+\mathrm{G}(\mathrm{d}, \mathrm{p})$ & 839 & 3.34 & 0.16 \\
$(9)$ & $\mathrm{C}_{18} \mathrm{H}_{36} \mathrm{O}_{2}$ & aug-cc-pVDZ & 784 & 3.38 & 0.46 \\
\hline
\end{tabular}

${ }^{a}$ The number of basis functions ( $N_{\mathrm{b} a s}$ ) is listed for comparison. MAXD stands for the maximum absolute deviation and MAD means the mean absolute deviation of all $3 N$ Cartesian gradient components. 
Table 4.

Comparison of the CIM-MP2 Gradients and Conventional MP2 Gradients (in $\mathrm{m} E_{\mathrm{h}} / \mathrm{a}_{0}$ ) for Five Structures of System (7) at the def2-SVP Basis Set

\begin{tabular}{ccc}
\hline entry & MAXD & MAD \\
1 & 0.45 & 0.06 \\
2 & 0.66 & 0.06 \\
3 & 0.52 & 0.06 \\
4 & 0.48 & 0.06 \\
5 & 0.56 & 0.06 \\
\hline
\end{tabular}



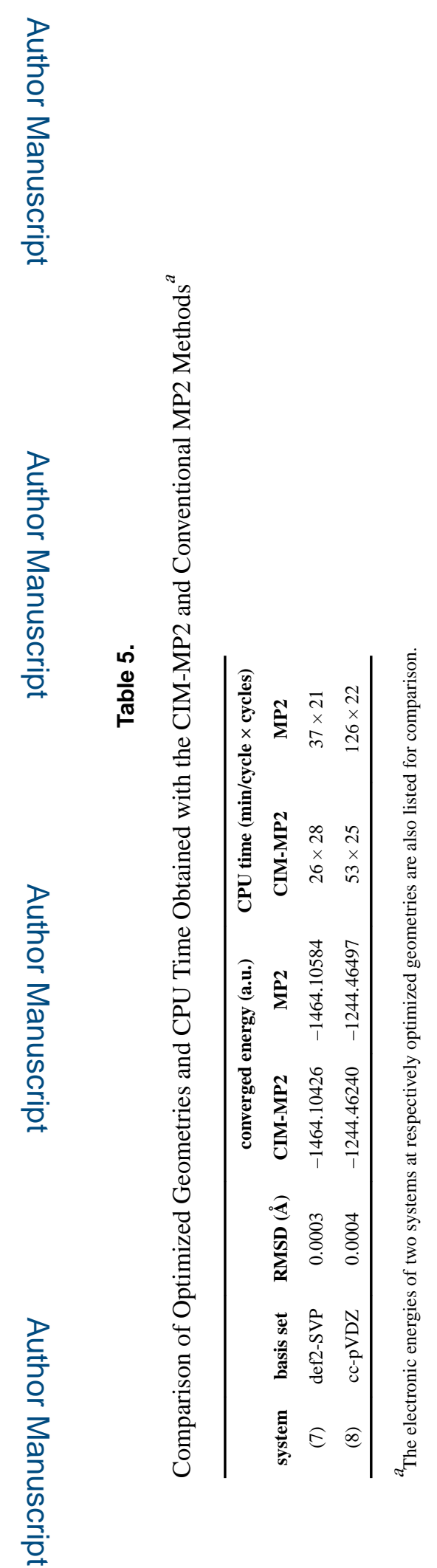

J Chem Theory Comput. Author manuscript; available in PMC 2020 June 11. 
Table 6.

Comparison of the Parameters of the Nine Hydrogen Bonds Obtained with Three Different Methods for Z$(\mathrm{Aib})_{11}-\mathrm{O} t \mathrm{Bu}^{a}$

\begin{tabular}{|c|c|c|c|c|c|c|c|}
\hline & \multicolumn{4}{|c|}{$\mathbf{N} \cdots \mathbf{O}$} & \multicolumn{3}{|c|}{ H $\cdots O$} \\
\hline & CIM-MP2 & M06-2X & B3LYP-D3 & crystal & CIM-MP2 & M06-2X & B3LYP-D3 \\
\hline 1 & 2.93 & 2.95 & 2.93 & 3.06 & 1.93 & 1.95 & 1.93 \\
\hline 2 & 2.99 & 3.00 & 2.97 & 2.89 & 1.97 & 2.00 & 1.97 \\
\hline 3 & 2.93 & 2.97 & 2.94 & 3.09 & 1.93 & 1.97 & 1.94 \\
\hline 4 & 2.93 & 2.97 & 2.94 & 3.01 & 1.93 & 1.97 & 1.93 \\
\hline 5 & 2.98 & 2.97 & 2.94 & 2.89 & 1.97 & 1.97 & 1.93 \\
\hline 6 & 2.94 & 2.98 & 2.94 & 3.01 & 1.94 & 1.98 & 1.94 \\
\hline 7 & 2.94 & 2.98 & 2.94 & 2.99 & 1.94 & 1.98 & 1.94 \\
\hline 8 & 3.02 & 2.99 & 2.97 & 3.00 & 2.02 & 2.00 & 1.98 \\
\hline 9 & 2.99 & 2.98 & 2.95 & 2.97 & 1.99 & 1.99 & 1.95 \\
\hline MAD & $0.08^{b}$ & $0.06^{b}$ & $0.07^{b}$ & & $\begin{array}{l}0.03^{c} \\
0.02^{d}\end{array}$ & & \\
\hline
\end{tabular}

a Bond lengths are in $\AA$.

${ }^{b}$ MAD with respect to the crystal structure.

$c$ MAD with respect to M06-2X structure.

${ }^{d}$ MAD with respect to B3LYP-D3 structure. 